FNUSAL - 2/95

\title{
A Consistent Study of the the Low Energy Baryon Spectrum and the Nucleon-Nucleon Interaction within the Chiral Quark Model
}

\author{
A. Valcarce ${ }^{(1)}$, P. González ${ }^{(2)}$, F. Fernández ${ }^{(1)}$ and V. Vento ${ }^{(2)}$ \\ (1) Grupo de Física Nuclear \\ Universidad de Salamanca, E-37008 Salamanca, Spain \\ (2) Departamento de Física Teórica e Instituto de Física Corpuscular \\ Universidad de Valencia-C.S.I.C, E-46100 Burjassot, Valencia, Spain
}

\begin{abstract}
By solving the Schrödinger equation for the three-quark system in the hyperspherical harmonic approach, we have studied the low energy part of the nucleon and $\Delta$ spectra using a quark-quark interaction which reproduces the nucleon-nucleon phenomenology. The quark-quark hamiltonian considered includes, besides the usual one-gluon exchange, pion and sigma exchanges generated by the chiral symmetry breaking. The baryonic spectrum obtained is reasonable and the resulting wave function gives consistency to the ansatz used in the two baryon system.
\end{abstract}


An exciting challenge in contemporary nuclear physics is to achieve a unified description of the baryon spectrum and the baryon-baryon interaction. Models have been widely used to study the properties of the hadron spectrum due to the impossibility to solve Quantum Chromodynamics (QCD) at the current moment. In particular, the quark potential models incorporate the perturbative one-gluon exchange quark-quark $(q q)$ potential $\left(V_{\mathrm{OGE}}\right)$ derived from QCD as well as a parametrization of some nonperturbative effects through a $q q$ confining potential $\left(V_{\text {con }}\right)$ [1]. Such an effective theory [the quark-gluon coupling constant is taken as an effective one and the constituent quark masses $\left(m_{q}\right)$ are parameters fitted from the baryon magnetic moments] provides a reasonable understanding of the baryonic spectrum and the baryon properties [2]. The adequate description of the low energy baryonic spectrum comes in part from the fact that the OGE interaction has an appropriate spin-isospin dependence which is responsible for the hyperfine splitting (e.g. $\Delta-\mathrm{N}$ mass difference).

The idea of an interquark potential has been also used to study baryon-baryon interactions. Actually, the repulsive core of the nucleon-nucleon (NN) force has been shown to arise from the color-spin structure of the $V_{\mathrm{OGE}}$ [3]. Nevertheless, the same scheme has been proven incapable of describing the long range part of the NN interaction unless pion exchange between quarks is introduced. From the basic theory, the origin of the one-pion exchange potential $\left(V_{\mathrm{OPE}}\right)$ is associated with the spontaneous chiral symmetry breaking of QCD. Moreover, the inclusion of the $q q$ sigma potential $\left(V_{\mathrm{OSE}}\right)$ consistently with its chiral partner (the pion) allows also to reproduce the intermediate range NN interaction as well as the deuteron properties [四,

The consistent description of both scenarios (baryon spectrum and nucleon-nucleon interaction) in a model based on interactions between quarks is still an open problem. There are different spectroscopic models in the literature [2] that through a fit of the parameters (sometimes an independent fit for the positive and negative parity sectors) are able to describe the low-energy baryon spectrum. Other quark models have been successfully used to reproduce the nucleon-nucleon interaction [3]. Certainly, to judge critically a model one should study the predictions in both sectors. 
We start from a $q q$ interaction whose parameters are determined studying the two nucleon properties and charge exchange reactions, and then, we proceed to analyze the baryon spectrum for this interaction. As we shall discuss, the predictions for the baryon masses and the baryon wave functions are not only a stringent test of the interaction but also a consistency test of the ansatz wave function used to generate the NN interaction in the resonating group method (RGM) calculations.

The quark-quark potential is explicitly given by [4],

$$
V_{q q}\left(\vec{r}_{i j}\right)=V_{\text {con }}\left(\vec{r}_{i j}\right)+V_{O G E}\left(\vec{r}_{i j}\right)+V_{O P E}\left(\vec{r}_{i j}\right)+V_{O S E}\left(\vec{r}_{i j}\right)
$$

where $\vec{r}_{i j}$ is the interquark distance $\left(\vec{r}_{i j}=\vec{r}_{i}-\vec{r}_{j}\right) . V_{O G E}$ is the one-gluon exchange potential with a smeared (of $r_{0}$ range) $\delta$ function term in order to avoid an unbound spectrum [6]:

$$
V_{O G E}\left(\vec{r}_{i j}\right)=\frac{1}{4} \alpha_{s} \vec{\lambda}_{i} \cdot \vec{\lambda}_{j}\left\{\frac{1}{r_{i j}}-\frac{1}{4 m_{q}^{2}}\left[1+\frac{2}{3} \vec{\sigma}_{i} \cdot \vec{\sigma}_{j}\right] \frac{e^{-r_{i j} / r_{0}}}{r_{0}^{2} r_{i j}}-\frac{1}{4 m_{q}^{2} r_{i j}^{3}} S_{i j}\right\}
$$

$\alpha_{s}$ is the effective quark-quark-gluon coupling constant and the $\lambda^{\prime} s$ are the $S U(3)$ color matrices. The $\sigma^{\prime} s$ stand for the spin Pauli matrices and $S_{i j}$ is the quark tensor operator $S_{i j}=3\left(\vec{\sigma}_{i} \cdot \hat{r}_{i j}\right)\left(\vec{\sigma}_{j} \cdot \hat{r}_{i j}\right)-\vec{\sigma}_{i} \cdot \vec{\sigma}_{j}$.

$V_{O P E}$ and $V_{O S E}$ are the one-pion and one-sigma exchange potentials given by:

$$
\begin{aligned}
V_{\mathrm{OPE}}\left(\vec{r}_{i j}\right)= & \frac{1}{3} \alpha_{c h} \frac{\Lambda^{2}}{\Lambda^{2}-m_{\pi}^{2}} m_{\pi}\left\{\left[Y\left(m_{\pi} r_{i j}\right)-\frac{\Lambda^{3}}{m_{\pi}^{3}} Y\left(\Lambda r_{i j}\right)\right] \vec{\sigma}_{i} \cdot \vec{\sigma}_{j}+\right. \\
& {\left.\left[H\left(m_{\pi} r_{i j}\right)-\frac{\Lambda^{3}}{m_{\pi}^{3}} H\left(\Lambda r_{i j}\right)\right] S_{i j}\right\} \vec{\tau}_{i} \cdot \vec{\tau}_{j} } \\
V_{\mathrm{OSE}}\left(\vec{r}_{i j}\right)= & -\alpha_{c h} \frac{4 m_{q}^{2}}{m_{\pi}^{2}} \frac{\Lambda^{2}}{\Lambda^{2}-m_{\sigma}^{2}} m_{\sigma}\left[Y\left(m_{\sigma} r_{i j}\right)-\frac{\Lambda}{m_{\sigma}} Y\left(\Lambda r_{i j}\right)\right]
\end{aligned}
$$

where $m_{\pi}\left(m_{\sigma}\right)$ is the pion (sigma) mass, $\alpha_{c h}$ is the chiral coupling constant related to the $\pi N N$ coupling constant by $\alpha_{c h}=\left(\frac{3}{5}\right)^{2} \frac{g_{\pi N N}^{2}}{4 \pi} \frac{m_{\pi}^{2}}{4 m_{N}^{2}}, \Lambda$ is a cutoff parameter, and $Y(x), H(x)$ are the Yukawa functions defined as:

$$
Y(x)=\frac{e^{-x}}{x} \quad, \quad H(x)=\left(1+\frac{3}{x}+\frac{3}{x^{2}}\right) Y(x)
$$


With this interaction, the two baryon system has been studied in the framework of the RGM. For the spatial part of the wave function of the quarks, a harmonic oscillator ground state was assumed,

$$
\eta_{\mathrm{os}}\left(\vec{r}_{i}-\vec{R}\right)=\left(\frac{1}{\pi b^{2}}\right)^{3 / 4} e^{-\left(\vec{r}_{i}-\vec{R}\right)^{2} / 2 b^{2}}
$$

where $\vec{R}$ is a parameter which determines the position of the baryon and $b$ is the harmonic oscillator constant.

Using a unique set of parameters, the NN scattering phase shifts [4], the static and electromagnetic properties of the deuteron [5] and the charge-exchange reaction $p p \rightarrow n \Delta^{++}$ [7] have been well reproduced. In fact, the OPE and OSE provide the medium and long-range behavior required to reproduce the NN phenomenology. Additionally, the pure nucleon- $\Delta$ interaction has the correct properties as indirectly derived from the $\pi N N$ system $\mathbb{8} \|$. Let us note, that even reproducing all these observables there is no constraint on the radial structure of the confining potential, since the baryon-baryon interaction does not depend on it [9].

To evaluate the baryonic spectrum with the just described potential $\left(V_{c o n}+V_{O G E}+V_{O P E}+\right.$ $\left.V_{O S E}\right)$ we have solved exactly the Schrödinger equation in the hyperspherical harmonic approach [10], this is by expanding the three-quark wave function in terms of a set of basis functions on the hypersphere unity (of dimension 6, the number of independent coordinates). For the potentials we make use of, the truncation of the series (for the positive parity states we have included the first two terms in the hyperspherical expansion, $\mathrm{k}=0$ and $\mathrm{k}=2$, and for the negative parity states only the $\mathrm{k}=1$ term) does not suppose any significant loss of precision [11]. As we have no coupling to continuum channels our states do not exhibit resonance features.

As stated above, the radial structure of the confinement cannot be fixed from the NN interaction. However, the baryon spectrum strongly depends on the confining potential. In fact, the widely used quadratic potential places the Roper resonance $1 \mathrm{GeV}$ above the ground state (if the strength is fixed variationally as in RGM), while it appears in the correct 
position if a linear potential is used. This fact favors a linear form for the confinement $\left[V_{\text {con }}\left(\vec{r}_{i j}\right)=-a_{c}\left(\vec{\lambda}_{i} \cdot \vec{\lambda}_{j}\right) r_{i j}\right]$ as suggested by meson spectroscopy and lattice calculations [12], to which we shall restrict from now on. The values of the parameters are taken from Ref. [5] and quoted in Table I, and the predicted $N$ and $\Delta$ spectra appear in Figure 1.

It is worth to note that the chiral pseudoscalar interaction contributes (together with the OGE) to the hyperfine splitting, driving the value of $\alpha_{s}$ to $0.4-0.5$, close to the QCD one. On the other hand, the $(\vec{\sigma} \cdot \vec{\sigma})(\vec{\tau} . \vec{\tau})$ structure of the pion interaction gives attraction for symmetric spin-isospin pairs and repulsion for antisymmetric ones (a quite distinctive feature since the color-magnetic part of the OGE gives similar contributions in both cases). This lowers the position of the first nucleon radial excitation [Roper resonance, $N(1440)$ ] with regard to the first negative parity state solving part of the discrepancy between usual two-body potential models (the predicted relative energy positions of the Roper and the first negative parity appear inverted) and experiment. To have the same effect (quasidegeneration of the first positive and negative parity excitations) in a model without pion interaction, the OGE exchange Coulomb interaction has to be very much strengthed [11]. A complete inversion as required by the experimental data can be achieved just by a small modification of the values of the pion interaction constants. For instance, using $\Lambda=5.0 \mathrm{fm}^{-1}$ instead of $\Lambda=4.2 \mathrm{fm}^{-1}$ one obtains $516 \mathrm{MeV}$ for the excitation energy of the Roper resonance and $534 \mathrm{MeV}$ for the first negative parity excited state. Nevertheless this modification would destroy the agreement in the NN system.

Quantitatively, the relevant role played by the pion interaction to this respect is shown in Table II where the results with the full potential [ $N(1440)$ and $N^{*}(1535)$ almost degenerated] are compared to the ones obtained when the pion interaction is removed and the OGE strength is fitted to the experimental $\Delta-N$ mass difference. As can be seen in this table, with the suppression of the pionic interaction, the $N^{*}(1535)$ appears deeply bound with respect to the Roper resonance.

Regarding the negative parity states of the nucleon and $\Delta$, they appear almost degenerated due to the potential we use. The only difference comes from the small contribution 
of the pion tensor interaction, which depends on the total angular momentum $J$. On the other hand, the splitting among the negative parity states is insufficient as compared to data. This might be an indication of the need of fine tuning the spin-spin interaction (and correspondingly a refitting of the parameters in the NN sector) as well as a signature of the possible relevance of spin-orbit terms, not considered here. Let us point out that due to the value of our coupling constant $\alpha_{s}$ and to the fact that the Galilei invariant spin-orbit force of the one-sigma exchange cancels the corresponding term of the one gluon exchange [13] the spin-orbit strength is very much reduced as compared to other models [2] where its inclusion would destroy the overall fit of the spectrum. Although a refinement of the potential incorporating a spin-orbit contribution has been recently proposed [13] the existence of Galilei non-invariant terms, spin-orbit force associated to confinement, relativistic corrections at the same order,... prevent making any definitive statement about how relevant the spin-orbit interaction should be. As we are interested in in the consistent treatment of the one and two body problems, we do not proceed further along this direction and center our attention in the examination of the wave functions.

In Figure 2 we compare the wave function we obtain for the nucleon ground state with the ansatz used in the RGM formalism for three different values of the oscillator parameter b. Although at long range all the wave functions look very similar, the discrepancy at the origin reaches a factor four. This effect may be very important for quantities depending on the value of the wave function at small distances. However, is not clear the importance of this discrepancy in the evaluation of the phase shifts. From the technical point of view a complete RGM calculation would be very complicated to perform with the full solution of the Schrödinger equation, but one can easily compare the diagonal kernels of the NN potential (which correspond to the diagonal part of those used in Refs. [4,5] to calculate the phase shifts and the deuteron properties) with both types of wave functions as shown in Figure 3. We see how the NN potential calculated with the full solution of the Schrödinger equation looks pretty similar to the potential calculated with the harmonic oscillator wave function with $b=0.518 \mathrm{fm}$, this is precisely the value used in the RGM calculation of the 
phase shifts and deuteron properties conferring to it a self-consistent character. Other values of $b$ give quite different potentials even in the medium-long range. In this way, the validity of the gaussian ansatz wave function in the RGM calculations is confirmed, and the old controversy about the possible values of the parameter $b$ can be solved.

As a summary, we have analyzed the baryonic low energy spectrum with a potential model that includes, besides the usual one-gluon exchange, pion and sigma exchanges between quarks as dictated by the breaking of chiral symmetry. It is worth to notice that all the parameters of the interaction are fixed to reproduce the two nucleon phenomenology and none of them is fixed to the spectrum.

The predicted baryon spectrum up to $0.7 \mathrm{GeV}$ of excitation energy is reasonable, and a linear form of the confinement is preferred. The pion interaction favors the correct relative position of the positive and negative parity states. Baryon wave functions are also obtained and compared to the ansatz considered in previous studies of the baryon spectrum, nucleonnucleon phase shifts and deuteron properties. The difference in the short range behavior suggests the possible relevance of a reanalysis of the one-body properties calculated with gaussian ansatzs. Concerning the long-range part, the ansatz gaussian wave function is valid for the study of the two body properties and we can figure out the value of the harmonic oscillator parameter, $b$, to be consistently used in two-nucleon conventional calculations.

Although much work is needed along this direction (strange sector, decay process, etc.) we think that the proposed model could serve as a good starting point to achieve a unified description of the spectrum and the baryon-baryon interaction.

\section{ACKNOWLEDGMENTS}

This work has been partially supported by Dirección General de Investigación Científica y Técnica (DGICYT) under the Contract No. PB91-0119, by CICYT grant AEN 93-0234 and by EU project ERBCHBICT941800. 


\section{REFERENCES}

[1] A. de Rújula, H. Georgi and S.L. Glashow, Phys. Rev. D 12 (1975) 147.

[2] N. Isgur and G. Karl, Phys. Rev. D 18 (1978) 4187; D 19 (1979) 2653, [Erratum, Phys. Rev. D23 (1981) 817]; D 20 (1979), 1191.

B. Silvestre-Brac and C. Gignoux, Phys. Rev. D 32 (1985) 743.

M. Gianini, Rep. Prog. Phys. 54 (1991) 453.

[3] M. Oka and K. Yazaki, Prog. Theor. Phys. 66 (1980) 556, 572; Phys. Lett. B 90 (1980) 41.

A. Faessler, F. Fernández, G. Lübeck and K. Shimizu, Phys. Lett. B 112 (1982) 201; Nucl. Phys. A 402 (1983) 555.

[4] F. Fernández, A. Valcarce, U. Straub and A. Faessler, Jour. of Phys. G 19 (1993) 2013.

[5] A. Valcarce, A. Buchmann, F. Fernández and A. Faessler, Phys. Rev. C 50 (1994) 2246.

[6] R.K. Bhaduri, L.E. Cohler and Y. Nogami, Phys. Rev. Lett. 44 (1980) 1369.

[7] F. Fernández, A. Valcarce, P. González, and V. Vento, Phys. Lett. B 287 (1992) 35; Nucl. Phys. A 567 (1994) 741.

[8] A. Valcarce, Ph.D. Thesis, University of Salamanca, (1993).

A. Valcarce, F. Fernández, H. Garcilazo, M.T. Peña, and P.U. Sauer, Phys. Rev. C 49 (1994) 1799.

[9] K. Shimizu, Rep. Prog. Phys. 52 (1989) 1.

[10] J. L. Ballot and M. Fabre de la Ripelle, Ann. Phys. (NY) 127 (1980) 62.

[11] B. Desplanques et al., Z. Phys. A 343 (1992) 331.

[12] F. Gutbrod and I. Montway, Phys. Lett. B 136 (1984) 411.

[13] A. Valcarce, A. Buchmann, F. Fernández and A. Faessler, Phys. Rev. C 51 (1995) 1480. 
[14] Particle Data Group, Phys. Rev. D 50 (1994) 1173. 


\section{FIGURES}

FIG. 1. Relative energy nucleon and delta spectrum up to $0.7 \mathrm{GeV}$ excitation energy. The solid line corresponds to the predictions of our model with a linear confining potential. The shaded region, whose size stands for the experimental uncertainty, represents the experimental data [14].

FIG. 2. Main radial component of the nucleon wave function in terms of the hyperradius $\rho$. The solid line corresponds to the solution of the Schrödinger equation. The long, medium and

short-dashed lines to gaussian ansatzs with values of the parameter $b=0.4,0.518,0.6$ respectively.

FIG. 3. Diagonal kernels of the NN potential for the channel $(\mathrm{S}, \mathrm{T})=(1,0)$ and for different nucleon wave functions. The solid line corresponds to the solution of the Schrödinger equation. The long, medium, and short-dashed lines to gaussian ansatzs with values of the parameter $b=0.4,0.518,0.6$ respectively. 


\section{TABLES}

TABLE I. Quark model parameters.

\begin{tabular}{cc}
\hline \hline$m_{q}(\mathrm{MeV})$ & 313 \\
$\alpha_{s}$ & 0.485 \\
$a_{c}\left(\mathrm{MeV} \cdot \mathrm{fm}^{-1}\right)$ & 91.488 \\
$\alpha_{c h}$ & 0.0269 \\
$r_{0}(f m)$ & 0.0367 \\
$m_{\sigma}\left(f m^{-1}\right)$ & 3.42 \\
$m_{\pi}\left(\mathrm{fm}^{-1}\right)$ & 0.7 \\
$\Lambda\left(f m^{-1}\right)$ & 4.2 \\
\hline \hline
\end{tabular}

TABLE II. Relative energy $(\mathrm{MeV})$ of the Roper and the first negative parity nucleon excitations.

\begin{tabular}{ccc}
\hline \hline & $N(1440)$ & $N^{*}(1535)$ \\
\hline$V=V_{\text {con }}+V_{O G E}+V_{O P E}+V_{O S E}$ & 530 & 512 \\
$V=V_{\text {con }}+V_{O G E}+V_{O S E}$ & 521 & 365 \\
\hline \hline
\end{tabular}


This figure "fig1-1.png" is available in "png" format from: http://arxiv.org/ps/nucl-th/9509009v2 
This figure "fig1-2.png" is available in "png" format from: http://arxiv.org/ps/nucl-th/9509009v2 
This figure "fig1-3.png" is available in "png" format from: http://arxiv.org/ps/nucl-th/9509009v2 